
\title{
25 Research Square \\ Title: Does the use of 3D Cones give a chance to postpone the use of resection prosthesis?
}

\section{Daniel Kotrych}

Department of Paediatric Orthopaedics and Oncology, Pomeranian Medical University, Szczecin

Adam Brodecki

Specialist Orthopedic and Rehabilitation Hospital "Gorka"

Sławomir Marcinkowski

Specialist Orthopedic and Rehabilitation Hospital "Gorka"

Marcin Anuszkiewicz

Specialist Orthopedic and Rehabilitation Hospital "Gorka"

Jakub Kleszowski

Specialist Orthopedic and Rehabilitation Hospital "Gorka"

Dawid Ciechanowicz ( $\nabla$ dciechanowicz@edu.pum.edu.pl)

Department of Paediatric Orthopaedics and Oncology, Pomeranian Medical University, Szczecin Andrzej Bohatyrewicz

Pomeranian Medical University

\section{Research Article}

Keywords: Total knee arthroplasty, revision knee arthroplasty, 3D printed Cone augments, metaphyseal bone loss, AORI 3

Posted Date: May 19th, 2021

DOI: https://doi.org/10.21203/rs.3.rs-495933/v1

License: (c) (1) This work is licensed under a Creative Commons Attribution 4.0 International License.

Read Full License 


\section{Abstract}

Background and purpose: Revision procedures and the resulting bone loss are still a big challenge for orthopedic surgeons. Therefor we checked, what post-operative functional outcomes 3D printed Cone augments offer in patients with bone defects (AORI classification type 2 and 3 ) of the knee joint and does use of Cones can delay the necessity to use resection prothesis?

Methods Data from 64 patients $(M=22 ; W=42)$ who underwent a total knee arthroplasty (TKA) revision in two clinical hospitals were included in the retrospective analysis. 3D printed Cones were used in all patients. The Knee Society Clinical Rating System (KSS) and the range of motion in the knee joint were used for the functional assessment of patients.

Results The mean follow-up was 22 months (range, 12 to 38 months). Survival for aseptic loosening at follow-up was $100 \%$. Infection occurred in $2(3.1 \%)$ patients. The mean KSS score increased from 12.75 points preoperatively to 66.56 points postoperatively $(p<0.001)$. While the mean range of motion in the knee joint changed from 61.15 degrees preoperatively to 115.93 degrees postoperative $(p<0.001)$.

Conclusion Due to the good functional results of patients and the low rate of complications, 3D printed Cone augments seems to be a good solution for patients requiring a TKA revision procedure. When used in patients with bone defects classified as $2 \mathrm{~B}$ and 3 (AORI), they can be a good alternative, delaying the need for resection prostheses.

\section{Introduction}

Total knee arthroplasty is an effective and commonly used method of treatment of advanced osteoarthritis. All over the world, the number of primary arthroplasty procedures is constantly growing. In 2005 a total of 523.000 joint replacement operations were performed in the US. It is estimated that this number will increase by $673 \%$ to 3.480 .000 procedures in 2030 in US. The number of revisions in the same year amounted to 38.500 and is expected to increase by over $600 \%$ to 268.200 in 2030 in US [1, 2]. The most common reason of revision are infections, instability, wrong size and fixation of implants. In the case of late revisions ( $>2$ years from the primary procedure), these are: polyethylene wear (34\%) and aseptic loosening (24\%) [3].

The decision to perform a total knee arthroplasty revision should be considered in many aspects, especially in terms of the expected results. The procedure is associated with a lot of problems. The most important are the reconstruction of bone defects, obtaining a stable and correct components fixation, restoring the joint line, determining the correct traction of the patella, obtaining uniform tension of the collateral ligaments and identical space in the flexion-extension movement $[4,5]$. The survival of implants after revision surgery is lower ( $86 \%$ over 10 years) compared to primary surgery ( $95 \%$ over 15 years) [6]. This is due to a more complex clinical situation, mainly related to bone loss and change in bone density. 
The treatment of bone defects has evolved over the last years. Various strategies have been described, mainly depending on the size of the bone defect, including the use of cement, metal augments or bone graft [7]. While the treatment of minor and moderate defects shows good results, treatment methods for major bone defects remain problematic [8]. Especially in the metaphyseal zone. High hopes can be pinned on the use of Cone-type augments, made of porous surfaces, enabling biological integration through bone ingrowth [9]. The aim of this study is to check the clinical results in patients treated surgically with 3D printed metal Cone augments and in particular to determine whether the use of metaphyseal augments in patients with type 2 and 3 defects according to AORI is a chance to delay the use of resection procedures.

\section{Results}

Demographic data The study group $(n=64)$ consisted of 42 women $(66 \%)$ and 22 men $(34 \%)$. The mean age of the patients at the time of surgery was 71 years (range, 57 to 80 years). The mean follow-up was 22 months (range, 12 to 38 months). In the analyzing group it was the first revision surgery for 41 (64.1\%) patients. 12 (18.8\%) patients had two previous operations, 4 (6.2\%) patients had three, and 7 (10.9\%) patients had four previous operations. In $35(54.7 \%)$ cases, the reason for revision was incorrect rotation of the endoprosthesis components and pain, loosening in $18(28.1 \%)$ cases, infection in $9(14.1 \%)$ and polyethylene wear in $2(3.1 \%)$ cases. Patients operated on due to infection underwent a two-stage procedure. Duration between stages was on average 6 weeks (Table 1). 
Table 1

Demographic data of patients reconstructed with custom-made 3D printed Cone Triathlon TS

\begin{tabular}{|lll|}
\hline Data & Patients & Percentage (\%) \\
\hline Age and gander & 71 years (range, 57-80) & \\
Age (mean years) & & $34 / 66$ \\
\hline Gender (male/female) & $22 / 42$ & 64.1 \\
\hline Revision surgery & 41 & 18,8 \\
First & 12 & 6,2 \\
Second & 4 & 10,9 \\
Third & 7 & \\
Fourth & 35 & 54.7 \\
Reason of revision & 18 & 28.1 \\
Incorrect components rotation & 9 & 14.1 \\
Loosening & 2 & 3.1 \\
Infection & & \\
Polyethylene wear & & \\
\hline
\end{tabular}

Operational outcomes For the reconstruction of massive bone defects, 3D printed Cone Triathlon TS (Stryker, Mahwah, New Jersey, USA) was used, including: 38 (59.4\%) tibial part (13 symmetrical and 25 asymmetrical) and $26(40.6 \%)$ femoral part. In 13 (20.3\%) cases Cones augmentation involved both the femur and the tibia.

Based on the AORI classification, $65.7 \%$ of tibial defects $(n=25)$ and $7.7 \%$ of the femoral defects $(n=2)$ were classified as type $2 \mathrm{~B}$ using pre-operative radiographs, computer tomography and intraoperative evaluation, while $34.2 \%$ of the tibial bone loss $(n=13)$ and $92.3 \%$ of the femoral bone loss $(n=24)$ were classified as type 3.

On the tibia side 64 stems were used, including 8 (12.5\%) fixed with cement. On the femoral side 64 stems were used, all uncemented. The length of tibial stems were $100 \mathrm{~mm}$ in $49(76.6 \%) \mathrm{knees}, 50 \mathrm{~mm}$ in 8 (12.5\%) and $150 \mathrm{~mm}$ in 7 (10.9\%) knees. On the femoral side, all stems $100 \mathrm{~mm}$ long. In one case, a patella prosthesis was implanted. Radiographic analysis showed bone-cone integration in all cases at the $98 \%$ of the border bone area. (Table 2 ). 
Table 2

Summary of operational outcomes in study group, treated with custom-made 3D printed Cone Triathlon TS $(n=64)$.

${ }^{1}$ cem. - cemented; ${ }^{2}$ uncem. - uncemented

\begin{tabular}{|lll|}
\hline Data & Patients & Percentage (\%) \\
\hline 3D printed Cone & 38 & 59.4 \\
Tibial part & & \\
\hline Femoral part & 26 & 40.6 \\
Both & 13 & 20.3 \\
\hline Bone defects (AORI) & 27 & 42.2 \\
Type 2B & 37 & 57.8 \\
Type 3 & & \\
Type of fixation & $8 / 56$ & $12.5 / 87.5$ \\
Tibia (cem. ${ }^{1}$ /uncem. ${ }^{2}$ ) & $0 / 64$ & $0 / 100$ \\
Femur (cem. ${ }^{1}$ / ${ }^{2}$ (uncem. ${ }^{2}$ ) & & \\
\hline Length of the tibial stems & 8 & 12.5 \\
50mm & 49 & 76.6 \\
100mm & 7 & 10.9 \\
150mm & & \\
\hline Length of the femoral stems & 64 & 100 \\
100mm & & \\
\hline
\end{tabular}

Complications Survival for aseptic loosening at follow-up (22 months) was 100\% (95\% Cl $=95-100 \%)$. Deep infection (S. aureus) occurred in $2(3.1 \%)$ patients 6 weeks after implantation. In our group, a total of $14(21.9 \%)$ patients underwent additional operations. The most common treatments are irrigation and debridement. No intraoperative complications related to Cone implantation were reported.

Functional results The mean preoperative range of motion in the knee was $61.14545( \pm 6.059636)$ degrees. After surgery, increased by an average of 54.78 points $(p<0.001)$ to $115.9273( \pm 6.624462)$ degrees. The opening mean score in the 100-degree KSS questionnaire was $12.74545( \pm 7.506092)$ points, after the procedure increased by an average of 66.56 points $(p<0.001)$ to $79.32727( \pm 3.288896)$ points (Fig. 1). 
Our study has limitations related to a retrospective analysis carried out on a heterogeneous group of patients. However the effects of the retrospective nature of the study are mitigated by the prospective collection of data. It is worth adding that this is one of the few studies of a series of cases with the use of 3D Cones in a large number of patients undergoing functional assessment. The use of implants was determined by surgeons with no preferred indication of one type. Another limitation is the imperfection of the KSS scale as a measure of clinical outcome that combines subjective outcomes with implant survival data. However, it is worth adding that it is the most frequently used classification for functional assessment of patients after TKA. Finally, the length of the observation is relatively short. However, 3D printing Cone implants were introduced in Poland in 2017. Therefore, further monitoring of our population will provide additional information.

Revision procedures after TKA are often a big challenge for surgeons. Additional problems arise especially when large bone defects occur ( 2 and 3 according to the AORI classification). According to our experience and the available literature, the key to the success of the procedure is appropriate planning, which takes into account e.g. zonal classification system. It determines three levels of implant fixation: joint surface, metaphysis and diaphysis [10]. Most revision systems achieve stability due to the distribution of loads on the joint surface and diaphysis zones, however it is in the metaphyseal zone that the greatest forces occur, the uneven distribution of which leads to loosening [11]. Understanding the role of fixation zones allows to create a methodology of conduct. The components should be firmly seated with proper distribution of mechanical forces, which is a decisive success factor. Lack of uniform load leads to movements exceeding $150 \mu \mathrm{m}$, which stimulates the formation of soft tissue, preventing implant osteointegration [12].

Methods of treating bone defects have been described in detail in the literature, especially in the short and medium-term follow-up period. Smaller, closed defects can be treated with a bone substitute or bone cement. Larger with bolt-reinforced cement or modular metal parts. The joint surface defects require the use of structural grafts or metal implants [5]. The evolution of materials has contributed to the development of porous surfaces that improve mechanical stability. To provide structural and biomechanical reconstruction of the metaphyseal area, titanium implants can be used to increase the surface area and support the remaining implants. These solutions include Sleeve's and Cone's. They are two different technologies with different philosophies. In our practice, we decided to use Cones for several reasons. One of them is the independence of zone implantation, which enables the distribution of forces beyond the articular area, protecting it from excessive stresses. The fixation in the metaphyseal zone is independent of the joint surface and diaphysis zones. Ensuring the independence of the positioning of the tibial tray and the extension allows for $>85 \%$ of the adjustment of the stem to the bone shaft without disturbing the mechanical axis [13]. In other cases, the fit can be achieved using a cemented stem. Another reason is the covering of the implant modeled on the structure of the trabecular bone. An average porosity of $80 \%$ combined with a modulus of elasticity ( $3 \mathrm{GPa}$ ), low stiffness and a high coefficient of friction increase osteoconductive, ensuring physiological load transfer and reducing the risk of loosening [14]. A great advantage of Cones is the low potential of bacterial adhesion and high 
survival [15]. Bonanzing et al. conducted a meta-analysis of 432 implanted Cones with an average followup of 42 months. Features of loosening were shown by $1.15 \%$ [16].

The change in bone density (on average by $27 \%$ ) after primary arthroplasty, correlated with its severe loss and it is a serious challenge in TKA revision [14]. 3D-printed titanium Cones, with anatomical shapes and porous structure can provide structural support while offering the potential for permanent biological fixation. The use of structural grafts has been associated with long remodeling times, limited graft availability, nonunion or resorption. Admittedly, Sandiford et al. proved comparable effectiveness in the application of both solutions, however, most reports indicate a high revision rate exceeding even $20 \%$ (over a 10-year period) [17] The first generation of Cones showed promising clinical and radiological results in TKA revisions. Their limitation was imprecise bone preparation and limited availability of sizes and shapes. The second generation offers a much wider range and safer preparation. [18]

The production technique based on 3D printing allowed the creation of a wide range of sizes for high fit, based on the use of a large CT database to determine the location and shape for optimal bone coverage and support [19]. The tools adapted to the size significantly reduced the risk of uncontrolled milling without obtaining a guarantee of geometric fit and the need to use cement. Faizan et al. compared traditional and 3D printed implants. In the case of using traditional implants, the incidence of intraoperative fractures during preparation was $4 \%$. By analyzing movements during valgus and simulated steps, the research showed similar values for the use of central tibial Cones for both technologies. In the case of asymmetrical tibial and femoral cones, they were much smaller compared to traditional [20].

The benefits of using an implant based on 3D printing were described by Patel et al., Assessing the stability and radiological results from the third to the sixth month after surgery as $100 \%$ [21]. Denehy et al. Performed a multicenter review of 62 TKA revisions using 3D Cones, with at least two years of followup. There were no cases of aseptic loosening and the survival rate was $90.2 \%$ [19]. In both studies the reason for the review was infections. The authors noted no signs of loosening in radiological examinations or progressive lines translucent to X-rays(after excluding patients with infection).

In our study the mean score in KSS questionnaire improved from 12.5 to 79.3 points. Similar results were reported by Girerd [22]. Tetreault also showed $98 \%$ survival over a 2-year period. The authors observed four cases of unsuccessful osseointegration [23]. Divano et al. Reported $100 \%$ aseptic survival in the mean 5 years and $2 \%$ revision due to infection, treated without the need for Cone removal. At the same time authors indicated $96 \%$ survival of the first generation femoral Cone in a series of 159 cases, but with a $24 \%$ fracture rate. The complication rate for 3D Cones was $2.1 \%$ [24]. A potential failure may be the use of Cones in sclerotic bone, which is a serious obstacle to osteointegration, especially on the tibia side where the risk of loosening is greatest [14, 18, 23]. You JS et. all presented no deep infection in 17 cases with a mean follow-up of 3.5 years [25]. Our study also reported a very low rate of infections -2 cases $(1.2 \%)$ within the first 6 weeks after surgery. 
So far, severe bone defects, classified as type 3 according to AORI, were most often treated with the use of a structural graft or resection prostheses. By using the multi-zone fixation technique, we believe that Cones can also be effectively used in such cases. Their use allows to raise the level of implant fixations, ensuring the correct reconstruction of the joint line, while avoiding further bone resection and sharing the load forces between the metaphyseal and join surface zones. Such an application is described by Kukrejaa et al. presenting a series of 6 cases (AORI type 3) using the tibial Cones. Radiographic evaluation showed $100 \%$ of osteointegration recorded on final radiographs in all patients after a mean follow-up of 4.1 years [27]. Similarly, Meneghini et al. described the use of Cone implants in the reconstruction of the tibia and joint line as an effective solution for delaying the use of a respectable endoprosthesis [28]. Our experience also shows this possibility. In 9 cases, we reconstructed the metaphyseal zone of the tibia using the asymmetrical Cone with total reconstruction of the condyle. In 4 cases, we used the asymmetric Cone to rebuild the tibial surface, resigning from the use of a tibial augment. This decision was dictated by osteoporotic bone tissue and the belief in a more favorable, superficial force distribution into the diaphisis zone. In 3 patients, the broken bone fragments with ligament attachments were based on the femoral Cone and in one case reinforced with titan wire loops. The radiological evaluation of the image over a 2-year period did not reveal any signs of loosening. Physical examination confirmed ligamentous stability with a range of motion of $0-120$. In one case, we used the femoral and tibial cones to fill the defects after removing the loosened sleeves, and in one case after removing the hinged prosthesis, which effectively allowed to fill the defects classified as AORI 3 type. We also believe that the use of Cones allows to reduce the need to use resection prosthesis in doubtful cases. Their use allows to rebuild destroyed metaphyseal zone and to restore the joint line. However, in the case of significant bone loss, reaching diaphysis, we recommend the use of resection prostheses with fixation in the bone shaft.

Conclusion The use of Cone implants has gained importance in the revision of the knee joint due to its biological and mechanical properties. Structural stiffness and stability due to the high coefficient of friction provide a structural reinforcement in the weaker spongy bone of the metaphyseal zone, ensuring the correct distribution of mechanical forces. Their use could reducing the risk of infection. The available literature and our experience confirm the assumption that Cones is a real option in the effective treatment of cavities classified according to $\mathrm{AORI}$ as type $2 \mathrm{~B}$ and 3 . Summarizing the available data, we conclude that in the short term 3D printed Cones provide good strengthening of the metaphyseal zone. These results are encouraging, although they must be confirmed by longer observation.

\section{Methods}

Between January 2017 and January 2020 the number of 64 knee arthroplasty revisions were performed with use of the 3D printed Cone augments. Data was collected from two clinical hospitals. Due to the retrospective nature of the scientific work the Bioethics Committee at the Pomeranian Medical University in Szczecin has waived the ethical approval. All patients or their relatives gave written informed consent to be included in scientific studies at the admission to the hospital. All procedures performed in studies involving human participants were in accordance with the ethical standards of the institutional and/or 
national research committee and with the 1964 Helsinki declaration and its later amendments or comparable ethical standards. Qualification for surgery was undertaken independently by two orthopedic specialists (D.K. and S.M) experienced in total knee arthroplasty (TKA) revision procedures. The decision on the choice of stem length and method of fixation was made by the surgeons based on planning and intraoperative evaluation. The surgeons mentioned above also performed all surgeries.

Patients were reviewed retrospectively. The following factors were assessed: the reason for the revision and the number of previous operations, the number of complication (infections, implant loosening, fracture), integration of the implant with bone tissue (signs of loosening defined as implant migration or radioactivity $>=2 \mathrm{~mm}$ along the entire component). In addition, it was evaluated the functional assessment of the joint, using The Knee Society Clinical Rating System (KSS). The evaluation was conducted pre-operatively and post-operatively in the 12th month after the surgery .

The sizes of the components used and the method of fixation were also checked. Bone tissue defects were assessed on the basis of preoperative radiological examinations ( $C T, X$-ray) according to the Anderson Orthopedic Research Institute (AORI) classification, which is one of the most commonly used scales for assessing bone defects. All radiographs were reviewed by five independent orthopedists (D.K., S.M., M.A., J.K., A.B.).

The results of the KSS were statistically analyzed. After checking the data distribution (The Shapiro-Wilk test), the student's T-test was performed in relation to the results in the questionnaire and to the range of motion in the knee joint. A p value $<0.05$ was considered as statistically significant difference. Statistical analysis was carried out in the Statistica 13 program.

\section{Declarations}

\section{Competing interests}

All authors declare no competing interests. The authors did not receive support from any organization for the submitted work. The authors have no conflicts of interest to declare that are relevant to the content of this article.

\section{Author contributions}

$D K, A B$, and SM designed the study. DK., SM., MA., JK .screened the medical record for patient inclusion. $A B$ and $D C$ collected the patient data. $D C$ and JK conducted the data analysis. AB, DK and DC drafted the manuscript. All authors reviewed and revised the manuscript.

\section{Acknowledgements}

None

\section{References}


1. Hamilton, D.F., Howie, C.R., Burnett, R., Simpson, A.H. \& Patton, J.T. Dealing with the predicted increase in demand for revision total knee arthroplasty: challenges, risks and opportunities. Bone Joint J. 97-B(6):723-8. doi: 10.1302/0301-620X.97B6.35185 (2015)

2. Kurtz, S., Ong, K., Lau, E., Mowat, F. \& Halpern, M. Projections of primary and revision hip and knee arthroplasty in the United States from 2005 to 2030. J Bone Joint Surg Am. 89(4):780-5. doi: 10.2106/JBJS.F.00222 (2007)

3. Pitta, M., et al. Failure After Modern Total Knee Arthroplasty: A Prospective Study of 18,065 Knees. J Arthroplasty. 407-414. doi: 10.1016/j.arth.2017.09.041 (2018)

4. van Kempen, R.W., Schimmel, J.J., van Hellemondt, G.G., Vandenneucker, H. \& Wymenga, A.B. Reason for revision TKA predicts clinical outcome: prospective evaluation of 150 consecutive patients with 2years followup. Clin Orthop Relat Res. 471(7):2296-302. doi: 10.1007/s11999-013-2940-8 (2013)

5. Lei, P.F., Hu, R.Y. \& Hu, Y.H. Bone Defects in Revision Total Knee Arthroplasty and Management. Orthop Surg.11(1):15-24. doi: 10.1111/os.12425 (2019)

6. Liang, $\mathrm{H}$., et al. Comparison of mode of failure between primary and revision total knee arthroplasties. Orthop Traumatol Surg Res.104(2):171-176. doi: 10.1016/j.otsr.2017.10.003 (2018)

7. Panegrossi, G., et al. Bone loss management in total knee revision surgery. Int Orthop.38(2):419-27. doi: 10.1007/s00264-013-2262-1 (2014)

8. Gill, U.N., Ahmed, N., Noor, S.S., Memon, I.A. \& Memon, Z.A. Management of the bone loss by metaphyseal sleeves in primary and revision knee arthroplasty: clinical experience and outcome after forty three cases. Int Orthop.44(11):2315-2320. doi: 10.1007/s00264-020-04663-1 (2020)

9. Sculco, P.K., Abdel, M.P., Hanssen, A.D. \& Lewallen, D.G. The management of bone loss in revision total knee arthroplasty: rebuild, reinforce, and augment. Bone Joint J.98-B(1 Suppl A):120-4. doi: 10.1302/0301-620X.98B1.36345 (2016)

10. Morgan-Jones, R., Oussedik, S.I., Graichen, H. \& Haddad, F.S. Zonal fixation in revision total knee arthroplasty. Bone Joint J. 97-B(2):147-9. doi: 10.1302/0301-620X.97B2.34144 (2015)

11. Vasso, M., Beaufils, P., Cerciello, S. \& Schiavone Panni, A. Bone loss following knee arthroplasty: potential treatment options. Arch Orthop Trauma Surg. 134(4):543-53. doi: 10.1007/s00402-0141941-8 (2014)

12. Parfitt, A.M. Targeted and nontargeted bone remodeling: relationship to basic multicellular unit origination and progression. Bone.30(1):5-7. doi: 10.1016/s8756-3282(01)00642-1 (2002)

13. Bédard, M., Cabrejo-Jones, K., Angers, M., Pelletier-Roy, R. \& Pelet, S. The Effect of Porous Tantalum Cones on Mechanical Alignment and Canal-Fill Ratio in Revision Total Knee Arthroplasty Performed with Uncemented Stems. J Arthroplasty. 30(11):1995-8. doi: 10.1016/j.arth.2015.05.016 (2015)

14. Jensen, C.L., Petersen, M.M., Schrøder, H.M. \& Lund, B. Bone mineral density changes of the proximal tibia after revision total knee arthroplasty. A randomised study with the use of porous tantalum metaphyseal cones. Int Orthop.36(9):1857-63. doi: 10.1007/s00264-012-1601-y (2012)

15. Zhang, X.M., et al. Ta-Coated Titanium Surface With Superior Bacteriostasis And Osseointegration. Int J Nanomedicine. 14:8693-8706. doi: 10.2147/IJN.S218640 (2019) 
16. Bonanzinga, T., et al. Are Trabecular Metal Cones a Valid Option to Treat Metaphyseal Bone Defects in Complex Primary and Revision Knee Arthroplasty? Joints.14;6(1):58-64. doi: 10.1055/s-00371608950 (2017)

17. Sandiford, N.A., Misur, P., Garbuz, D.S., Greidanus, N.V. \& Masri, B.A. No Difference Between Trabecular Metal Cones and Femoral Head Allografts in Revision TKA: Minimum 5-year Followup. Clin Orthop Relat Res.475(1):118-124. doi: 10.1007/s11999-016-4898-9 (2017)

18. Zanirato, A., et al. Metaphyseal cones and sleeves in revision total knee arthroplasty: Two sides of the same coin? Complications, clinical and radiological results-a systematic review of the literature. Musculoskelet Surg. 104(1):25-35. doi: 10.1007/s12306-019-00598-y (2020)

19. Denehy, K.M., et al. Metaphyseal Fixation Using Highly Porous Cones in Revision Total Knee Arthroplasty: Minimum Two Year Follow Up Study. J Arthroplasty. 34(10):2439-2443. doi: 10.1016/j.arth.2019.03.045 (2019)

20. Faizan, A., et al. Development and Verification of Novel Porous Titanium Metaphyseal Cones for Revision Total Knee Arthroplasty. J Arthroplasty. 32(6):1946-1953. doi: 10.1016/j.arth.2017.01.013 (2017)

21. Patel, N.K., et al. Highly Porous Metaphyseal Cones in Revision Total Knee Arthroplasty: A Case Series. J Knee Surg. 29(8):614-620. doi: 10.1055/s-0036-1593369 (2016)

22. Girerd, D., et al. Total knee arthroplasty revision with trabecular tantalum cones: Preliminary retrospective study of 51 patients from two centres with a minimal 2-year follow-up. Orthop Traumatol Surg Res.102(4):429-33. doi: 10.1016/j.otsr.2016.02.010 (2016)

23. Tetreault, M.W., Perry, K.I., Pagnano, M.W., Hanssen, A.D. \& Abdel, M.P. Excellent two-year survivorship of 3D-printed metaphyseal cones in revision total knee arthroplasty. Bone Joint J.102-

B(6_Supple_A):107-115. doi: 10.1302/0301-620X.102B6.BJJ-2019-1544.R1 (2020)

24. Divano, S., et al. Porous metal cones: gold standard for massive bone loss in complex revision knee arthroplasty? A systematic review of current literature. Arch Orthop Trauma Surg. 138(6):851-863. doi: 10.1007/s00402-018-2936-7 (2018)

25. You, J.S., et al. Addressing large tibial osseous defects in primary total knee arthroplasty using porous tantalum cones. Knee.26(1):228-239. doi: 10.1016/j.knee.2018.11.001 (2019)

26. Kamath, A.F., Lewallen, D.G. \& Hanssen, A.D. Porous tantalum metaphyseal cones for severe tibial bone loss in revision knee arthroplasty: a five to nine-year follow-up. J Bone Joint Surg Am.97(3):216-23. doi: 10.2106/JBJS.N.00540 (2015)

27. Kukreja, M.M. \& Swanson, T.V. Can tibial tantalum cones eventually eliminate the adjuvant use of metallic augments for AORI type 2B/3 metaphyseal defects??-A novel surgical technique and case series. Int J Surg Case Rep.53:200-206. doi: 10.1016/j.ijscr.2018.09.028 (2018)

28. Meneghini, R.M., Lewallen, D.G. \& Hanssen, A.D. Use of porous tantalum metaphyseal cones for severe tibial bone loss during revision total knee replacement. J Bone Joint Surg Am. 90(1):78-84. doi: 10.2106/JBJS.F.01495. PMID: 18171960. (2008) 
Figures

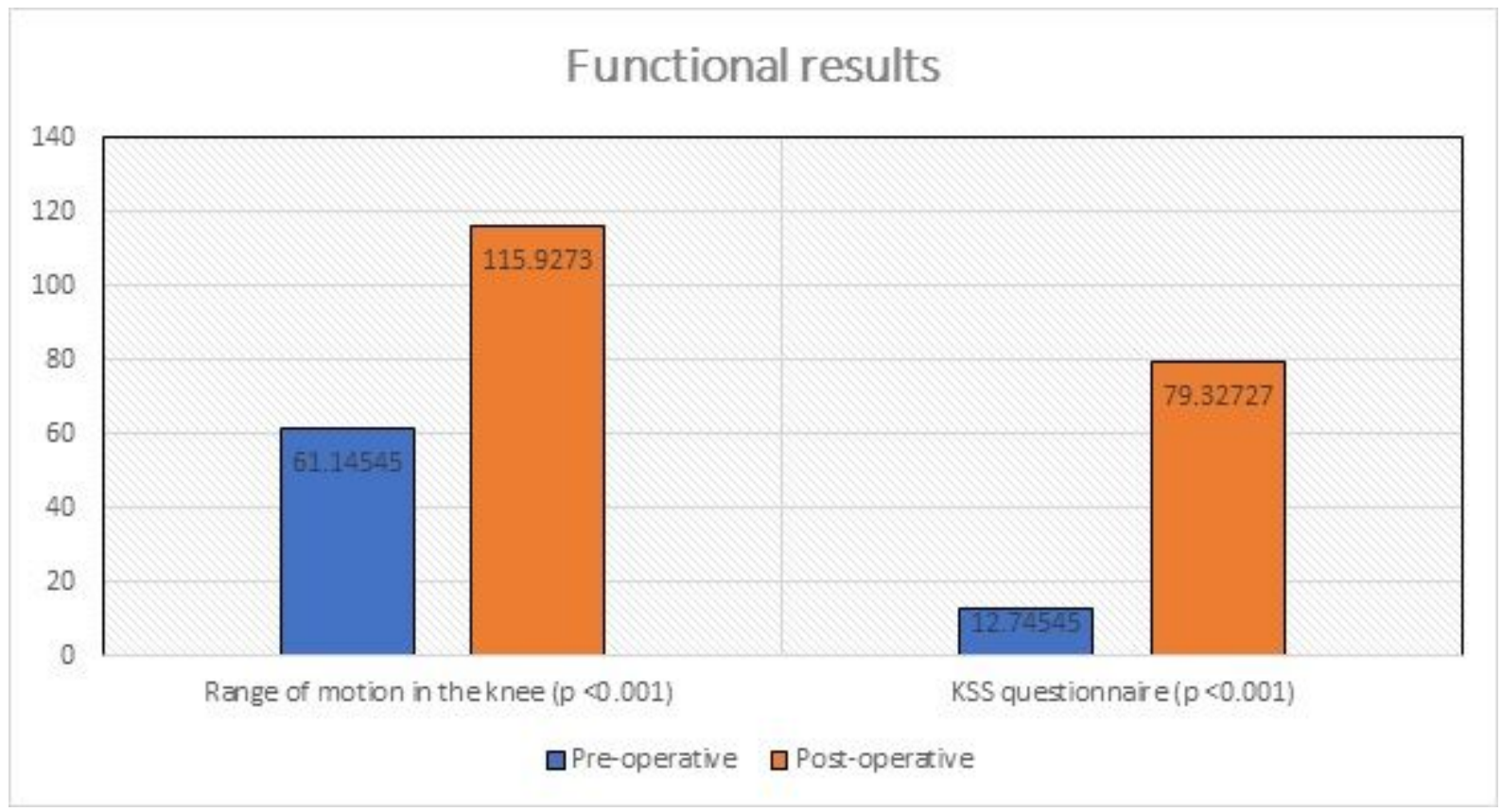

Figure 1

Comparison of patients' functional outcomes (KSS questionnaire and Range of motion) in the preoperative and 12 months postoperative period 